\title{
FMS wird Ende Juni 2004 aufgelöst
}

Prof. Dr. med. Reto Tscholl, Präsident FMS

Eine ausserordentliche Delegiertenversammlung der FMS hat am 8. Mai 2004 beschlossen, die Vereinigung per Ende Juni dieses Jahres aufzulösen. Die chirurgisch, interventionell und invasiv tätigen Ärzte sollen sich in einem neuen Dachverband zusammenschliessen.

Ursprünglich war geplant gewesen, die beiden Vereinigungen FMS und Union nach der Gründung eines gemeinsamen neuen Dachverbandes aufzulösen. Die Gründung des neuen Verbandes kam an der Versammlung vom 8. Mai 2004 jedoch nicht zustande. Eine neue Arbeitsgruppe soll bis September dieses Jahres die notwendigen Grundlagen für einen Dachverband ausarbeiten. Der Vorstand der FMS hat dennoch an dem mit der Union vereinbarten Fahrplan festgehalten und der ausserordentlichen Delegiertenversammlung vom 8. Mai die Auflösung der FMS beantragt. Die Mehrheit der Delegierten ist diesem Antrag gefolgt.

\section{Gründe für die Auflösung}

Welche Gründe haben den Vorstand der FMS dazu veranlasst, die Auflösung zu beantragen, bevor die Nachfolgeorganisation definitiv gegründet ist? Einerseits ist nur eine kurze Frist von einigen Monaten bis zur Neugründung des Dachverbandes im September 2004 zu überbrücken. Andererseits - und das war der entscheidende Grund - sind die wesentlichen Forderungen der FMS inzwischen von der FMH übernommen worden.

In der Schweizerischen Ärztezeitung hat der abtretende FMH-Präsident, Hans Heinrich Brunner, alle wesentlichen Positionen bestätigt, welche die FMS während Jahren im Zusammenhang mit der Einführung des TARMED vertreten hat [1]. Mit seiner Aussage, «dass der TARMED vor allem im Bereich KVG zu einem eigentlichen Exterminationsprogramm von operativ Tätigen bzw. Belegärzten auszuarten droht, verschärft noch durch die dauernde Taxpunktwertabsenkung, die nach sich langsam konkretisierender Erkenntnis vor allem jene treffen wird, die die Kostensteigerung gar nicht $\mathrm{zu}$ verantworten haben», bestätigt er vollumfänglich sämtliche Aussagen der FMS zum Thema TARMED. Und weiter schreibt er: «Es ist unbestritten, dass die Tätigkeiten des Belegarztes in den TARMEDTarifstrukturen nicht abgebildet sind - prosaisch formuliert: nicht adäquat bezahlt werden.»

Diese (wenn auch verspätete) Einsicht des FMH-Präsidenten ist erfreulich. Stossend ist hingegen die Behauptung, dass die FMH diese Position seit Jahren vertreten habe. Dass die FMH seit 1993 eine solche Haltung eingenommen hätte, ist reine Geschichtsklitterung. Es war ausschliesslich die von vielen angefeindete FMS, die den Behauptungen der FMH, alles sei bestens, was sich heute für jeden ersichtlich als falsch erwiesen hat, während Jahren entgegentrat und immer wieder davor warnte, dass die in grosser Zahl erbrachten operativen, radiologischen und sonstigen interventionellen Leistungen (sogenannte Routineeingriffe) nicht kostendeckend tarifiert wären. FMS:

Einige Zitate aus Medienmitteilungen der

- Die FMS verlangt, dass für folgende Bereiche noch eine vernünftige Regelung getroffen werden muss: Sockelzeiten, Assistenzen und Taxpunktwert. (29. August 2001)

- Belegärzte, die grundversicherte Patienten betreuen, werden entweder ihre Praxis schliessen müssen, oder sich auf die allein noch rentablen Grundleistungen der Praxis zurückziehen. (29. August 2001)

- Es wurde ein Tarifflickwerk genehmigt, das schon bei seiner Einführung überarbeitet werden muss, weil es ärztliche Fachgesellschaften existentiell gefährdet. (13. Dezember 2001)

Dadurch, dass der FMH-Präsident genau die von der FMS über Jahre verlangten Massnahmen jetzt zum Schutz der Radiologen und der Belegärzte ergreifen will, bestätigt er ungewollt, aber ausdrücklich, dass die FMS in ihrer Beurteilung der Folgen des Tarifs und somit in ihren Forderungen nach entsprechenden Korrekturen auf der ganzen Linie recht hatte. Einige der Kernanliegen der FMS sind damit erfüllt. Die FMS ist darüber sehr befriedigt, gleichzeitig aber auch erstaunt darüber, dass der FMH-Präsident offenbar meint, die Geschädigten hätten seine frühere ganz andere Position bereits vergessen oder würden ihm diese nachsehen. 
Wahl des FMH-Präsidenten ist Chance für Neuanfang

Die FMH-Wahlen vom 26. Juni 2004 geben der Ärzteschaft Gelegenheit, einen neuen, unverbrauchten Präsidenten zu wählen, der in der Lage sein wird, in einer Atmosphäre des Vertrauens einen Neuanfang zu machen. Unabhängig davon, wer als neuer FMH-Präsident gewählt wird, darf sich der rücksichtslose Umgang der FMH-Spitze mit den vitalen Interessen ganzer Ärztegruppen nicht wiederholen. Dies ist nur durch strukturelle Veränderungen der FMH zu erreichen, indem die FMH so umgebaut wird, dass nicht nur die Mehrheit der Grundversorger, sondern auch Minderheiten von Spezialisten in den FMH-Gremien adäquat repräsentiert sind und die Vertretung ihrer vitalen Interessen durch den Gesamtverband gesichert ist.
Falls die FMH-Spitze und später die Ärztekammer keine Hand zu einem solchen Umbau bieten, so wird die invasive Ärzteschaft entgegen ihrer eigentlichen Absicht gezwungen sein, ihren neuen Dachverband aus der FMH hinauszuführen.

\section{Support für TARMED-Klagen}

Rund 50 Schweizer Ärzte haben gegen die FMH und die Versicherer einen Prozess angestrengt, der die Ungültigkeit der TARMEDVerträge im Visier hat. Diese Klagen wurden von der FMS immer unterstützt. Der Support für die klagenden Ärzte ist auch nach der Auflösung der FMS gesichert - bis die Gerichte entschieden haben. 